\title{
Unusual intraorbital foreign body impactions, a case report
}

SADJ February 2019, Vol. 74 No. 1 p32 - p34

GP Mohotlhoane', M Mabongo²

\section{INTRODUCTION}

Foreign body impalements in the maxillofacial region are not uncommon, but intra-orbital foreign bodies (IOFBs) impactions are rare, the frequency being one in every six patients with orbital trauma. ${ }^{1}$ IOFBs can be classified into intra-ocular and extra-ocular foreign bodies, the latter being an object that lies within the orbit but outside the ocular globe. ${ }^{2}$ Intra-ocular foreign bodies can lead to globe rupture, infection, retinal toxicity, and vision loss, and thus detection is of the utmost importance, with imaging playing a key role. These foreign bodies (FB) pose a diagnostic challenge due to their size, difficulty of access and their close relation to vital structures. Ocular acuity and mobility should be investigated on admission as they are frequently associated with ocular trauma. ${ }^{2}$

IOFBs may result from trivial accidents, gunshot, industrial accidents, ${ }^{2}$ interpersonal violence e.g. knife impactions. When an impalement injury occurs in the craniofacial region it is usually referred to as Jael's Syndrome, based on the biblical story of a "nail" being hammered into the temple of a sleeping man (Judges IV, v.21). ${ }^{3}$

This article reports a case of a patient presenting with an orbito-infratemporal foreign body, (an extraocular foreign body that extends into the infratemporal fossa), resulting in restricted mouth opening. To our knowledge, impaction with this type of foreign body, a door handle, has not been previously reported.

\section{CASE REPORT}

A 34 year old male presented to Chris Hani Baragwanath Academic Hospital (CHBAH) with complaints of pain and irritation below the left eye. He gave a history of falling against a door, striking one side of his face five days prior to presentation. The door handle had been broken but the fractured piece had not been found. He had consulted his local clinic immediately after the injury with bleeding from the face in the infraorbital area, which was managed at the clinic, and he had then been referred to an ophthalmologist.

\section{Author affiliations:}

1. Gontse P. Mohotlhoane: BDS (UL), Registrar, Department of Maxillofacial and Oral Surgery, University of the Witwatersrand.

2. Mzubanzi Mabongo: STD (Unitra), B Dent (Medunsa), BDS Wits), MChD (MFOS) (UWC), Cert. Orofacial Pain (Rugter, USA), FCFS (Bangalore, India), Craniomaxillofacial Surgeon, Head of Clinical Unit, Department of Oral and Maxillofacial Surgery, University of Witwatersrand

Corresponding author: Mzubanzi Mabongo

Department of Oral and Maxillofacial Surgery, University of

Witwatersrand, Charlotte Maxeke Academic Hospital, Johannesburg,

South Africa. Email: mzubanzi.mabongo@wits.ac.za

\section{ACRONYM}

CHBAH: $\quad$ Chris Hani Baragwanath Academic Hospital

CT:

FBs:

IOFBs:

MRI:

OMFS: Computed Tomography Foreign Bodies Intra-orbital Foreign Bodies Magnetic Resonance Imaging Oral and Maxillofacial Surgery

The eye was assessed in the Ophthalmology Department at $\mathrm{CHBAH}$ a day after injury when no abnormalities were detected. The patient was discharged with analgesics and an ointment. He reported back to Ophthalmology after two days with severe pain and irritation and was then referred to the Oral and Maxillofacial (OMFS) Department for further management.

On clinical assessment a small healing scar, about $5 \mathrm{~mm}$. in length, was noted on the lower eyelid (Fig 1). The orbital rims were intact and non-tender on palpation. The globe of the eye was intact and vision was normal from both eyes. Eye movement was not restricted and there was no diplopia in all gazes. However there was slight pain on superior gaze. He also suffered a restricted mouth opening of $2.5 \mathrm{~cm}$ due to discomfort.

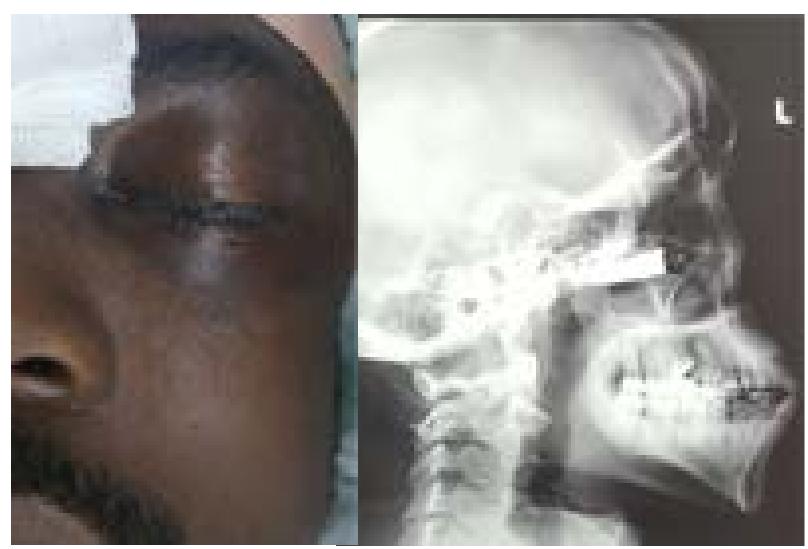

Figure 1 and 2. Pre-operative view and lateral skull view

Lateral and postero-anterior radiographic views of the skull were taken (Fig 2). On examination, a radiopaque object about $3 \mathrm{~cm}$ in length was evident in the infraorbital region. A computed tomography (CT) scan was requested to assess the extent of the injury and the tissues involved (Figs 2 and 3). The report indicated that there was a metal object in the orbit which was extending into the infra-temporal fossa through the inferior orbital fissure, but neither fractures nor vascular injuries were diagnosed. 


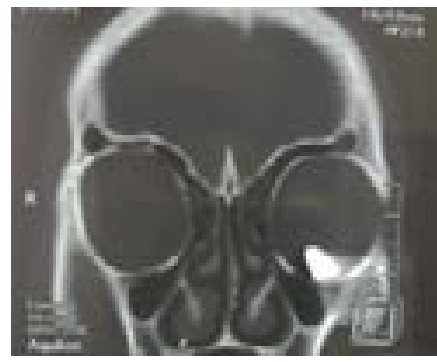

Figure 3. Coronal view of the CT-Scan indicating the door handle above the infra-orbital rim.

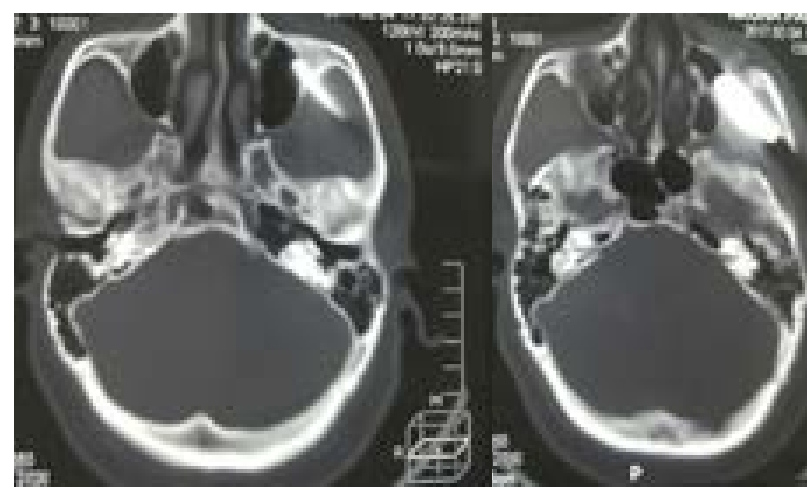

Figure 4. Axial views of the CT-Scan indicating door handle extending into the infra-temporal region.

A local anesthetic with a vasoconstrictor was injected in the area of the laceration prior to making an incision through the infraorbital scar, extending about $1 \mathrm{~cm}$. A careful dissection through the soft tissue layers was made, avoiding the application of any forces which may have displaced the object.

The tip of the door handle was encountered after dissection through the subcutaneous layer. The object was clamped with Crile forceps and gently removed, reversing along the path of penetration, which was judiciously followed to prevent further injuries (Figs 5 and 6)).

The area was thoroughly assessed for any fractures, and none were noted. The wound was debrided and irrigated with normal saline. The wound was sutured in layers with 3.0 vicryl rapid and 5.0 nylon for the skin.

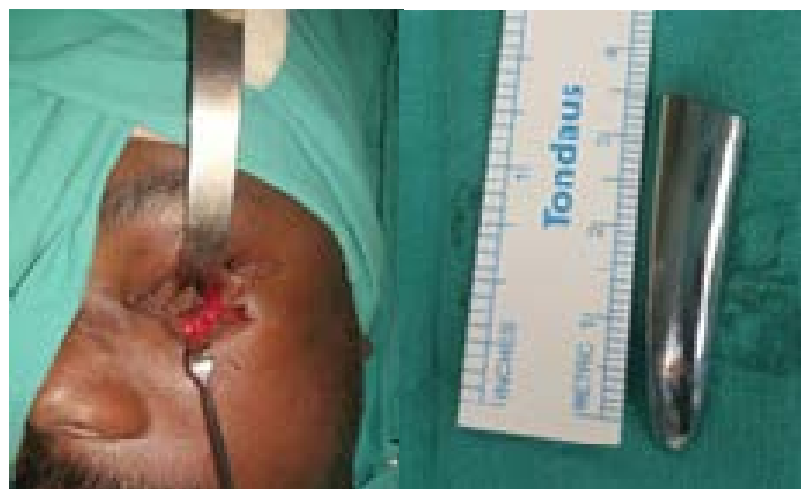

Figure 5 and 6 . Door handle being removed through the lower eyelid incision; the tip of the door handle after being removed

On review after a week the patient was asymptomatic, eye function was normal and could open the mouth wide, with an interincisal distance of $45 \mathrm{~mm}$. The nylon suture was removed and the patient was discharged.

\section{DISCUSSION}

Impalement of the orbit by a foreign body is a rare phenomenon. A prevalence of 0,29\% was reported in Nigeria. ${ }^{4}$ The foreign bodies that have been involved in craniofacial impaction may be classified according to their composition as metallic, such as steel; non-metallic, which may be organic, such as wood and vegetable matter and inorganic, such as glass. ${ }^{2}$

Prompt detection and accurate localization of IOFBs is essential for the optimum management of patients, enabling the surgeon to plan the most atraumatic method of removing the intruder. Unfortunately, the nature and extent of the injury in this case was not diagnosed on initial presentation, perhaps associated with there being only a minor laceration on the lower eyelid and oedema. Other factors that must have contributed would be an assessment that focused on the globe and the failure to take plain radiographs.

Clinical management of foreign bodies is dependent on their composition and the site of impalement. Intraocular foreign bodies are usually removed surgically to prevent complications from chemical reactions (e.g., siderosis from iron) or infection. Extraocular foreign bodies are managed conservatively, and therefore, it is important to accurately differentiate between intraocular and extraocular locations. Retained foreign bodies may give rise to cellulitis, abscess, fistulas, and impaired vision and motility. ${ }^{1}$

Diagnostic imaging modalities are critical in the diagnosis, confirmation and localization of IOFBs. ${ }^{5}$ Plain radiographs are inexpensive and readily available, but radiolucent material, such as graphite, plastic, and fresh or dry wood may be underestimated. ${ }^{1,3}$ In this patient the presence of a foreign body was diagnosed with routine radiology.

The clinical sign of restricted mouth opening could not be explained based on that diagnostic imaging, and hence the CT scan was requested in order to determine the posterior extent of the injury.

Ocular ultra-sonic scanning offers a higher sensitivity than plain radiographs for detecting all kinds of IOFBs and is accurate for intraocular foreign bodies. However, it is time consuming, operator dependent, and may be technically difficult in an open globe. ${ }^{1}$

Computed tomography is very useful in the determination of the size of foreign bodies and in localizing them as intraocular, extraocular, or retro-ocular. CT is generally considered the gold standard in the evaluation of IOFBs because it is safe to use with metallic IOFBs and is also able to diagnose orbital wall fractures and orbital sepsis with high accuracy. ${ }^{1,2}$

CT is accurate at detecting and localizing intraorbital metallic, glass, and stone foreign bodies. ${ }^{1}$ In this case, CT scans were able to show that the foreign body was protruding into the infratemporal space, suggesting that there may have been a form of injury to the temporalis muscle. That would explain the restricted mouth opening associated with the intraorbital foreign body in this patient. 
Magnetic Resonance Imaging (MRI) examination is of value when there is a high suspicion of IOFBs, especially wooden IOFBs with a negative CT scan. However, failure to detect a metallic foreign body before MRI may result in globe perforation and blindness should the object move in response to magnetic forces during imaging. ${ }^{1}$

Indication for removal may include a superficial location, protrusion through the skin, ${ }^{6}$ large size, interference with ocular function, infection, persistent inflammation, and communication with intracranial cavity and or paranasal sinuses. ${ }^{1}$

\section{CONCLUSION}

Trauma officers should always harbour a high index of suspicion for impacted foreign bodies until their presence is otherwise excluded by plain radiography. Also, nonresolving abscess around the scars of previous injury, in the face of clear plain $\mathrm{x}$-rays, should be an indication for advanced diagnostic imaging.

\section{References}

1. Moretti A, Laus M, Crescenzi D, Croce A. Peri-orbital foreign body: a case report. Journal of Medical Case Reports 2012, 26;6(1):91.

2. Pinto A, Brunese L, Daniele S, Faggian A, Guarnieri G, Muto M, Romano $L$. Role of computed tomography in the assessment of intraorbital foreign bodies. In : Seminars in Ultrasound, CT and MRI 2012;33 (5): 392-5.

3. de Santana Santos T, Avelar RL, Melo AR, de Moraes HH, Dourado $E$. Current approach in the management of patients with foreign bodies in the maxillofacial region. Journal of Oral and Maxillofacial Surgery 2011;69(9):2376-82.

4. Olusanya AA, Aladelusi TO, Olanloye OM. Experience with impacted foreign bodies in the maxillofacial region at a Nigerian teaching hospital. Journal of the West African College of Surgeons 2015;5(3):1-15.

5. Parke DW, Flynn HW, Fisher YL. Management of intraocular foreign bodies: a clinical flight plan. Canadian Journal of Ophthalmology/Journal Canadien d'Ophtalmologie 2013; 48(1):8-12.

6. Espaillat A, Enzer Y, Lipsky S. Intraorbital metallic foreign body. Archives of Ophthalmology 1998;116(6):824-5. 